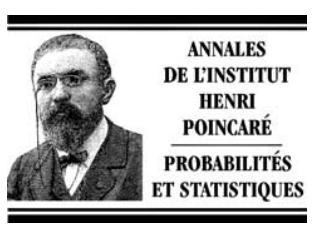

www.elsevier.com/locate/anihpb

\title{
Moments of passage times for Lévy processes
}

\author{
R.A. Doney ${ }^{a}$, R.A. Maller ${ }^{b, *, 1}$ \\ a Department of Mathematics, University of Manchester, Manchester M13 9PL, UK \\ b Centre for Financial Mathematics, MSI, School of Finance \& Applied Statistics, Australian National University, \\ Canberra, ACT, 0200 Australia \\ Received 12 February 2003; received in revised form 27 October 2003; accepted 4 November 2003 \\ Available online 21 February 2004
}

\begin{abstract}
We give necessary and sufficient conditions, in terms of characteristics of the process, for finiteness of moments of passage times of general Lévy processes above horizontal, linear or certain curved boundaries. They apply in particular to processes which drift almost surely to infinity, and lead to estimates of the rate of growth of certain expectations, constituting generalised kinds of renewal theorems. Further results concern the inverse local time at the maximum and the ladder height process, the amount of time spent below a given level, and the overall minimum of the Lévy process.
\end{abstract}

(c) 2004 Elsevier SAS. All rights reserved.

\section{Résumé}

Des conditions nécessaires et suffisantes sont données pour la finitude des moments de certains temps de passage des processus de Lévy.

(c) 2004 Elsevier SAS. All rights reserved.

MSC: primary 60J30, 60K05, 60F15, 60G40; secondary 60G10, 60G17, 60J65, 60J15

Keywords: Passage times of Lévy processes; Horizontal boundaries; Curved boundaries; Drift to infinity; Renewal theorems; Local time; Ladder height processes; Strong laws

\section{Introduction and main results}

Consider a Lévy process $X=\left\{X_{t}\right\}_{t} \geqslant 0$ with $E \mathrm{e}^{i \theta X_{t}}=\mathrm{e}^{t \Psi(\theta)}, t \geqslant 0$, where the Lévy exponent $\Psi$ is given by the Lévy-Khintchine formula [1, pp. 12-13]

$$
\Psi(\theta)=i \gamma \theta-\frac{1}{2} \sigma^{2} \theta^{2}+\int_{(-\infty, \infty)}\left(\mathrm{e}^{i \theta x}-1-i \theta x \mathbf{1}_{[-1,-1]}\right) \Pi(d x) .
$$

\footnotetext{
* Corresponding author.

E-mail addresses: rad@maths.man.ac.uk (R.A. Doney), ross.maller@anu.edu.au (R.A. Maller).

1 This work is partially supported by ARC Grant DP0210572.

0246-0203/\$ - see front matter (C) 2004 Elsevier SAS. All rights reserved.

doi:10.1016/j.anihpb.2003.10.005
} 
The quantities $\gamma, \sigma^{2}$ and $\Pi$ are called the characteristics of $X$. Eq. (1.1) is the analytic counterpart of the following standard decomposition (see [11, p. 120]): we have $X_{0}=0$ and

$$
X_{t}=\gamma t+\sigma B_{t}+X_{t}^{(1)}+X_{t}^{(2)},
$$

where $B$ is a standard Brownian motion, $X^{(1)}$ has Lévy exponent

$$
\Psi^{(1)}(\theta)=\int_{(-\infty, \infty)}\left(\mathrm{e}^{i \theta x}-1-i \theta x\right) \mathbf{1}_{[-1,-1]} d \Pi(x),
$$

and consequently has expectation 0 and finite moments of all orders, and $X^{(2)}$ is the compound Poisson process with Lévy exponent

$$
\Psi^{(2)}(\theta)=\int_{(-\infty, \infty)}\left(\mathrm{e}^{i \theta x}-1\right)\left(\mathbf{1}_{(1, \infty)}+\mathbf{1}_{(-\infty,-1)}\right) d \Pi(x) .
$$

Our main objects of study are the first and last passage times, defined by

$$
T_{r}=\inf \left\{t: X_{t}>r\right\}, \quad r>0
$$

(with $T_{r}=\infty$ if the set on the right-hand side is empty) and

$$
\Lambda_{r}=\sup \left\{t: X_{t} \leqslant r\right\}, \quad r \geqslant 0,
$$

and our main objective is to provide necessary and sufficient conditions, expressed in terms of the characteristics of $X$, for these random variables to have finite moments. These lead to kinds of generalised renewal theorems.

In random walk theory, $\tau_{0}$, the first increasing ladder epoch in a discrete time random walk, plays a key role in the study of first passage times, and is used in a crucial way in [8], which is our point of departure for the present paper. But $T_{0}$, the analogous object in the continuous time context, defined as in (1.5) (with $\left.r=0\right)$, in many cases of interest equals 0 almost surely (a.s.), and some of the methods of [8] fail. Thus our approach cannot simply be a matter of transferring the random walk results into the continuous time framework in a mechanical way. To bypass this difficulty we use an approach due to Doney [4] (see Proposition 8 below) which gives very convenient stochastic bounds for the process in terms of random walks. In our context the analogous result for $\tau_{0}$ is formulated by introducing the local time at the maximum, and an appropriate Lévy process version of the existence of moments of $\tau_{0}$ is in (1.17) below. For this purpose, let $L^{-1}=\left(L^{-1}(t), t \geqslant 0\right)$ denote the increasing ladder time process (inverse local time at the maximum) of $X$, with exponent $\kappa(q)=-\log E\left(\mathrm{e}^{-q L^{-1}(1)}\right)$. The increasing ladder height process will be denoted by $H$. See Bertoin [1], Chapter VI, for these.

To state our results we write, for $x>0, \bar{\Pi}^{+}(x)=\Pi((x, \infty)), \bar{\Pi}^{-}(x)=\Pi((-\infty,-x))$, and

$$
A(x)=\gamma+\bar{\Pi}^{+}(1)-\bar{\Pi}^{-}(1)+\int_{1}^{x}\left(\bar{\Pi}^{+}(y)-\bar{\Pi}^{-}(y)\right) d y,
$$

which is a kind of truncated mean; when $E\left|X_{1}\right|<\infty$ then $A(x) \rightarrow E X_{1}$ as $x \rightarrow \infty$. Let $X^{\#}=\left(-X_{t}, t \geqslant 0\right)$, and denote its Lévy measure by $\Pi^{\#}$. We will also need

$$
J_{-}=\int_{(1, \infty)}\left(\frac{x}{\bar{\Pi}^{+}(1)+\int_{1}^{x} \bar{\Pi}^{+}(y) d y}\right) \Pi^{\#}(d x)
$$

(when the denominator is positive; otherwise $J_{-}$need not be defined). This is relevant because it was shown in Doney and Maller [5] that $\lim _{t \rightarrow \infty} X_{t}=\infty$ a.s. if and only if

$$
J_{-}<\infty=\int_{1}^{\infty} \bar{\Pi}^{+}(y) d y \quad \text { or } \quad 0<E X_{1} \leqslant E\left|X_{1}\right|<\infty
$$


which complements the less explicit criterion (Bertoin [1, p. 167]), that

$$
\int_{1}^{\infty} t^{-1} P\left(X_{t} \leqslant 0\right) d t<\infty .
$$

[5] also shows that $\lim _{t \rightarrow \infty} X_{t} / t=\infty$ a.s. if and only if the first condition in (1.9) holds.

Since all our results are invariant under scaling, when $\Pi$ is not identically zero (so that $X$ does not reduce to Brownian motion) we can and will assume that $c_{1}=\bar{\Pi}^{+}(1)+\bar{\Pi}^{-}(1)>0$. Then we write (see (1.2))

$$
X_{0}^{(2)}=0, \quad X_{t}^{(2)}=\sum_{i=1}^{N_{t}} J_{i}, \quad t>0,
$$

where the $J_{i}$ are i.i.d. (independent and identically distributed) with

$$
P\left(J_{1} \in d x\right)=c_{1}^{-1}\left(\mathbf{1}_{(1, \infty)}+\mathbf{1}_{(-\infty,-1)}\right) \Pi(d x)
$$

and $\left\{N_{t}, t \geqslant 0\right\}$ is a Poisson process of rate $c_{1}$. The $J_{i},\left\{N_{t}, t \geqslant 0\right\}, X^{(1)}$ and $B$ are all independent of each other.

Our first task is to give a Lévy process version of Theorem 2.1 in [8].

Theorem 1. Fix $\alpha \geqslant 0$. The following are equivalent:

$$
\int_{1}^{\infty} t^{\alpha-1} P\left(X_{t} \leqslant r\right) d t<\infty \quad \text { for some (hence every) } r \geqslant 0 ;
$$

there is an $x_{0}>0$ such that $A(x)>0$ for $x \geqslant x_{0}$, and

$$
\begin{aligned}
& \int_{\left(x_{0}, \infty\right)}\left(\frac{x}{A(x)}\right)^{1+\alpha} \Pi^{\#}(d x)<\infty ; \\
& E T_{r}^{1+\alpha}<\infty \text { for some (hence every) } r>0 ; \\
& E \Lambda_{r}^{\alpha}<\infty \text { for some (hence every) } r \geqslant 0, \text { if } \alpha>0 ; \\
& P\left(\Lambda_{r}<\infty\right)=1 \text { for some (hence every) } r \geqslant 0, \text { if } \alpha=0 ; \\
& E\left(L^{-1}(1)\right)^{1+\alpha}<\infty .
\end{aligned}
$$

Remark. (i) By the definitions of $T_{r}$ and $\Lambda_{r},(1.15)$ is equivalent to

$$
\int_{0}^{\infty} t^{\alpha} P\left(\sup _{0 \leqslant s \leqslant t} X_{s} \leqslant r\right) d t<\infty \quad \text { for some (hence every) } r \geqslant 0 ;
$$

and the conditions in (1.16) are equivalent to

$$
\begin{aligned}
& \int_{0}^{\infty} t^{\alpha-1} P\left(\inf _{s \geqslant t} X_{s} \leqslant r\right) d t<\infty \quad \text { for some (hence every) } r \geqslant 0, \text { if } \alpha>0, \\
& \lim _{t \rightarrow \infty} P\left(\inf _{s \geqslant t} X_{s} \leqslant r\right)=0 \quad \text { for some (hence every) } r \geqslant 0, \text { if } \alpha=0 .
\end{aligned}
$$

(ii) Condition (1.13) implies (1.10), hence it implies $\lim _{t \rightarrow \infty} X_{t}=\infty$ a.s. Thus Theorem 1 deals only with processes that drift to $+\infty$ a.s., or equivalently, with processes satisfying (1.9). If the first condition in (1.9) holds it is easy to verify that $A(x) \sim \int_{1}^{x} \bar{\Pi}^{+}(y) d y \rightarrow \infty$ as $x \rightarrow \infty$, while $\lim _{x \rightarrow \infty} A(x)=E X_{1}>0$ if the second 
condition in (1.9) holds. (See also Lemma 13 below.) Now $\int_{1}^{\infty} x^{1+\alpha} \Pi^{\#}(d x)$ converges if and only if $E\left(X_{1}^{-}\right)^{\alpha+1}$ converges (Sato [11, p. 159]). Thus $A(x)>0$ for all large $x$ and (1.14) holds if and only if

$$
0<E X_{1} \leqslant E\left|X_{1}\right|<\infty \text { and } E\left(X_{1}^{-}\right)^{\alpha+1}<\infty
$$

or

$$
\int_{(1, \infty)}\left(\frac{x}{\int_{1}^{x} \bar{\Pi}^{+}(y) d y}\right)^{1+\alpha} \Pi^{\#}(d x)<\infty=\int_{1}^{\infty} \bar{\Pi}^{+}(x) d x .
$$

The next theorem gives a condition for $E T_{r}^{\alpha}$ to be finite when $0<\alpha<1$, and examines its rate of growth as $r \rightarrow \infty, 0<\alpha \leqslant 1$, when it is. Its proof will make use of the relation (Bertoin [1, p. 174])

$$
q \int_{0}^{\infty} \mathrm{e}^{-q t} P\left(T_{r}>t\right) d t=\kappa(q) V^{q}(r)
$$

where $r>0, q>0$, and

$$
V^{q}(r)=\int_{0}^{\infty} E\left(\mathrm{e}^{-q L^{-1}(t)} 1_{\left\{H_{t} \leqslant r\right\}}\right) d t .
$$

In what follows we will use $\asymp$ to mean that the ratio of two expressions is bounded below and above by two positive, finite constants for all sufficiently large $r$.

Theorem 2. (i) (No assumptions on X.) Let $0<\alpha<1$ and $r>0$. Then $E T_{r}^{\alpha}<\infty$ if and only if $q^{-\alpha-1} \kappa(q) \in$ $L(0,1)$, or, equivalently, if

$$
\int_{0}^{1} q^{-\alpha} \exp \left\{\int_{1}^{\infty} \mathrm{e}^{-q t} t^{-1} P\left(X_{t} \leqslant 0\right) d t\right\} d q<\infty .
$$

If this holds then

$$
\Gamma(1-\alpha) E T_{r}^{\alpha}=\alpha \int_{0}^{\infty} q^{-\alpha-1} \kappa(q) V^{q}(r) d q, \quad \text { for all } r>0 .
$$

(ii) If $\lim _{t \rightarrow \infty} X_{t}=+\infty$ a.s. (so that $A(x)>0$ for large enough $x$ ) then

$$
E T_{r} \asymp \frac{r}{A(r)}, \quad \text { as } r \rightarrow \infty \text {. }
$$

Condition (1.21) is not completely explicit but it can be used for example when $P\left(X_{t}<0\right) \rightarrow 1-\rho \in[0,1]$ as $t \rightarrow \infty$. Then it tells us that $E T_{r}^{\alpha}<\infty$ if $\alpha<\rho$ (as also follows when $0<\rho<1$ from Bertoin [1, p. 173]). In particular, if $X_{t} \stackrel{P}{\rightarrow} \infty$ as $t \rightarrow \infty$ then $E T_{r}^{\alpha}<\infty$ for each $\alpha<1$ (but $E T_{r}<\infty$ if and only if $X_{t} \rightarrow \infty$ a.s. as $t \rightarrow \infty$ ). If we assume more we can get a completely explicit solution, as in the next theorem.

Theorem 3. (i) For a fixed $\alpha>0$ for which the integral in (1.24) converges, we have $A(x)>0$ for all large $x$, and, as $r \rightarrow \infty$, 


$$
\begin{aligned}
& \int_{1}^{\infty} t^{\alpha-1} P\left(X_{t} \leqslant r\right) d t \asymp\left(\frac{r}{A(r)}\right)^{\alpha} ; \\
& E T_{r}^{\alpha} \asymp\left(\frac{r}{A(r)}\right)^{\alpha} ;
\end{aligned}
$$

and

$$
E \Lambda_{r}^{\alpha} \asymp\left(\frac{r}{A(r)}\right)^{\alpha} .
$$

(ii) Assume only that $\lim _{t \rightarrow \infty} X_{t}=\infty$ a.s. Then $A(x)>0$ for all large $x$ and

$$
\int_{1}^{\infty} t^{-1} P\left(X_{t} \leqslant r\right) d t \asymp \int_{1}^{\infty} t^{-1} P\left(\sup _{0 \leqslant s \leqslant t} X_{s} \leqslant r\right) d t \asymp \log \left(\frac{r}{A(r)}\right), \quad \text { as } r \rightarrow \infty \text {. }
$$

The next result adds to the equivalences in Theorem 1.

Theorem 4. Suppose $\lim _{t \rightarrow \infty} X_{t}=\infty$ a.s., so that $A(x)>0$ for all large $x$, and $\alpha>0$. Then (1.14) holds if and only if the following quantities have a finite moment of order $\alpha$ :

$$
\begin{aligned}
& T(-r) \mathbf{1}_{\{T(-r)<\infty\}}, \quad \text { where } T(-r)=\inf \left\{t: X_{t}<-r\right\}, r \geqslant 0 ; \\
& T_{\min }=\inf \left\{t: X_{t} \leqslant I_{\infty} \text { or } X_{t-} \leqslant I_{\infty}\right\}, \quad \text { where } I_{\infty}=\inf _{0<s<\infty} X_{s} ; \\
& O_{r}=\int_{0}^{\infty} \mathbf{1}_{\left\{X_{t}<r\right\}} d t, r \geqslant 0 .
\end{aligned}
$$

A further set of results in Proposition 4.1 of [8] gives a different, but related necessary and sufficient condition for some other quantities to have a finite moment of order $\alpha$. (See also [7].) To give the analogues for Lévy processes we need to introduce the process $H^{\#}=\left(H_{s}^{\#}, s \geqslant 0\right)$, which is the ladder height process for $X^{\#}$.

Theorem 5. Assume $\lim _{t \rightarrow \infty} X_{t}=\infty$ a.s., so that $A(x)>0$ for $x$ large enough, $x \geqslant x_{0}$, say, and fix $\alpha>0$. Then the following are equivalent:

$$
\begin{aligned}
& \int_{\left(x_{0}, \infty\right)}\left(\frac{x^{1+\alpha}}{A(x)}\right) \Pi^{\#}(d x)<\infty ; \\
& E\left(\left(H_{r}^{\#}\right)^{\alpha} ; H_{r}^{\#}<\infty\right)<\infty \quad \text { for some (hence every) } r>0 ; \\
& E\left(\left|I_{\infty}\right|^{\alpha}\right)<\infty ; \\
& E\left(\left|X_{T(-r)}\right|^{\alpha} ; T(-r)<\infty\right)<\infty \quad \text { for some (hence every) } r \geqslant 0 ; \\
& E\left(\sup _{0 \leqslant t \leqslant \Lambda_{r}}\left|X_{t}\right|^{\alpha}\right)<\infty \quad \text { for some (hence every) } r \geqslant 0 ; \\
& E\left(\sup _{0 \leqslant t \leqslant T(-r)}\left|X_{t}\right|^{\alpha} ; T(-r)<\infty\right)<\infty \text { for some (hence every) } r \geqslant 0 .
\end{aligned}
$$

Remark. (iii) In the case $0<E X_{1} \leqslant E\left|X_{1}\right|<\infty$, so that $\lim _{x \rightarrow \infty} A(x)=E X_{1}>0$, for all large $x$, (1.31) and (1.14) coincide, and reduce to $E\left(X_{1}^{-}\right)^{\alpha+1}<\infty$, but in general they are different. 
The next two theorems consider curved boundaries which are bounded above by concave (possibly linear) functions. Our method is to reduce the problem to one of constant boundaries, so Theorems 6 and 7 below can be considered as applications of the foregoing results. To formulate the curved boundary results, let $g(t)$ be a positive nondecreasing function on $[0, \infty)$, with $g(0)=0$, which is regularly varying with index $\rho$ as $t \rightarrow \infty$ (write this as $\left.g \in R V_{\rho}\right)$. Define

$$
T_{g}(r, a)=\inf \left\{t>0: X_{t}>r g(t+a)\right\}, \quad r>0, a>0 .
$$

Theorem 6. Fix $\alpha \geqslant 0$ and suppose $g \in R V_{\rho}$ with $0 \leqslant \rho<1$. Then $E\left(T_{g}(r, a)\right)^{1+\alpha}<\infty$ for some, hence every, $r>0, a>0$, if and only if $A(x)>0$ for all large $x$ and (1.14) holds.

Remark. (iv) The constant $a>0$ is introduced in (1.37) to keep the boundary away from 0 ; note that $T_{g}(r, a) \geqslant$ $T_{r g(a)}$ (with $T_{r}$ as in (1.5)). We need this because a Lévy process which has unbounded variation jumps immediately over a boundary which increases at a linear or slower than linear rate near 0, by virtue of a result of Rogozin [10] which states that, then,

$$
-\infty=\liminf _{t \rightarrow 0+} \frac{X_{t}}{t}<\limsup _{t \rightarrow 0+} \frac{X_{t}}{t}=+\infty \text { a.s. }
$$

A similar principle applies in the next result, which treats linear boundaries. Define $T_{1}(r, a)$ to be the version of $T_{g}(r, a)$ when $g(t)=t$.

Theorem 7. (i) $E\left(T_{1}(r, a)\right)<\infty$ for all $r>0$ and some $a>0$ if and only if

$$
J_{-}<\infty=\int_{1}^{\infty} \bar{\Pi}^{+}(x) d x
$$

(see (1.8) for $\left.J_{-}\right)$, and if this holds then, for each $\alpha \geqslant 0, E\left(T_{1}(r, a)\right)^{1+\alpha}<\infty$ for some, hence every, $r>0, a>0$, if and only if (1.19) holds.

(ii) $E\left(T_{1}(r, a)\right)<\infty$ for some but not all $r>0$, for some $a>0$, if and only if $0<E X_{1} \leqslant E\left|X_{1}\right|<\infty$, and if this holds then, for all $\alpha \geqslant 0, E\left(T_{1}(r, a)\right)^{1+\alpha}<\infty$ for some $r>0$, hence for all $0<r<E X_{1}$ and all $a>0$, if and only if $E\left(X_{1}^{-}\right)^{\alpha+1}<\infty$.

Remark. (v) Our methods do not give good estimates of the rate of growth of $E\left(T_{g}(r, a)\right)^{1+\alpha}$, as $r \rightarrow \infty$, in Theorems 6 and 7 (and in part (ii) of Theorem 7 we have $E\left(T_{1}(r, a)\right)^{1+\alpha}=\infty$ for $r \geqslant E X_{1}$, in any case).

We conclude this section with some examples of interest.

Example 1. Spectrally positive processes/subordinators. The characteristics satisfy $\gamma \in \mathbb{R}, \sigma^{2} \geqslant 0$ and $\Pi((-\infty, 0))=$ 0 , so $A(x)=\gamma+\bar{\Pi}^{+}(1)+\int_{1}^{x} \bar{\Pi}^{+}(y) d y$. We have $\lim _{t \rightarrow \infty} X_{t}=\infty$ a.s. if $\int_{1}^{\infty} \bar{\Pi}^{+}(x) d x=\infty$ or if $\int_{1}^{\infty} \bar{\Pi}^{+}(x) d x<\infty$ and $A(\infty)>0$. If either of these holds then $A(x)>0$ for all large $x$. So by Theorem 1 , in these cases, $E T_{r}^{1+\alpha}<\infty$ for all $\alpha \geqslant 0$ and $r>0$.

Example 2. Symmetric processes plus drift. These have $\bar{\Pi}^{+}(\cdot)=\bar{\Pi}^{-}(\cdot)$, so $A(x)=\gamma$. We have

$$
\int_{\left(x_{0}, \infty\right)} \frac{x \Pi^{\#}(d x)}{\int_{x_{0}}^{x} \bar{\Pi}^{+}(y) d y}=\int_{\left(x_{0}, \infty\right)} \frac{x \Pi(d x)}{\int_{x_{0}}^{x} \bar{\Pi}^{+}(y) d y}=\infty
$$

unless $\int_{1}^{\infty} \bar{\Pi}^{+}(x) d x<\infty$, in which case $E\left|X_{1}\right|<\infty$, and so by (1.9), we have $\lim _{t \rightarrow \infty} X_{t}=\infty$ a.s. if and only if $\gamma>0$. If this is so then $E T_{r}^{1+\alpha}<\infty$ if and only if $\int_{(1, \infty)} x^{1+\alpha} \Pi(d x)<\infty$, or, equivalently, $E\left|X_{1}\right|^{1+\alpha}<\infty$. 
Example 3. Jump diffusion processes are of the form

$$
X_{t}=a t+\sigma B_{t}+\sum_{j=1}^{N_{t}} Y_{j}
$$

where $a \in \mathbb{R}, N_{t}$ is a Poisson process of rate $c$, independent of the Brownian motion $B_{t}$, and of the $Y_{j}$, which are i.i.d. with c.d.f. $F(\cdot)$, also independent of $B_{t}$. Then $\bar{\Pi}^{+}(x)=c(1-F(x)), \bar{\Pi}^{-}(x)=c F(-x), x>0$, and

$$
\gamma=a+c \int_{[-1,1]} x d F(x)=a-c(1-F(1)-F(-1))+c \int_{0}^{1}(1-F(x)-F(-x)) d x .
$$

Using (1.7) we can easily check that

$$
A(x)=a+c \int_{0}^{x}(1-F(y)-F(-y)) d y=a+c A_{F}(x),
$$

say, where $A_{F}(x)=E\left(\left(Y_{1} \wedge x\right) \vee(-x)\right)$. Now $\lim _{t \rightarrow \infty} X_{t}=\infty$ a.s. is equivalent to (1.9), which in turn is equivalent to: $\sum_{1}^{n} Y_{i} / n \stackrel{\text { a.s. }}{\rightarrow} \infty$ as $n \rightarrow \infty$, or else $E\left|Y_{1}\right|<\infty$ and $a+c E Y_{1}=a+c A_{F}(\infty)>0$ [8]. These are equivalent to requiring that the random walk with steps $\tilde{Y}_{i}:=a+c Y_{i}$ drift to $\infty$ a.s. By Theorem 1 and Remark (ii) following it, $E T_{r}^{1+\alpha}<\infty$ for some $\alpha \geqslant 0$ if and only if one of the following holds: $E\left(Y_{1}^{-}\right)^{1+\alpha}<\infty$ (if $E\left|Y_{1}\right|<\infty$ and $\left.a+c E Y_{1}>0\right)$, or

$$
\int_{(1, \infty)}\left(\frac{x}{A_{F}(x)}\right)^{1+\alpha}|F(-d x)|<\infty
$$

(if $\sum_{1}^{n} Y_{i} / n \rightarrow \infty$ a.s.). By Theorem 2.1 of [8], this is equivalent to the finiteness of $E \tilde{T}_{r}^{1+\alpha}$, where $\tilde{T}_{r}=$ $\inf \left\{n \geqslant 1: \sum_{1}^{n} \tilde{Y}_{i}>r\right\}$.

Effectively, for the Jump Diffusion Process (Compound Poisson process when $a=\sigma=0$ ), conditions for the finiteness of the moments of passage times for the associated random walk transfer directly across to the same for the Lévy process, as we would expect.

Example 4 (Stable processes of order $v, 0<v<2$ ). For these, $\sigma=0, \bar{\Pi}^{+}(x)=c^{+} / x^{v}$ and $\bar{\Pi}^{-}(x)=c^{-} / x^{\nu}$, $x>0$, where $c^{+} \geqslant 0, c^{-} \geqslant 0$, and we allow a drift $\gamma \in \mathbb{R}$. If $c^{-}=0$ then we have Example 1 , so keep $c^{+} \geqslant 0$ and $c^{-}>0$. Now $\int_{1}^{\infty} \bar{\Pi}^{+}(x) d x=\infty$ if and only if $v \leqslant 1$, in which case

$$
J_{-} \asymp \int_{1}^{\infty} \frac{x}{x^{1-v}} \frac{d x}{x^{\nu+1}}=\infty
$$

so we can have a.s. drift to $\infty$ only if $E\left|X_{1}\right|<\infty$, equivalently, if $1<v<2$, and $E X_{1}>0$. The $A(\cdot)$ function then satisfies

$$
\lim _{x \rightarrow \infty} A(x)=\gamma+\lim _{x \rightarrow \infty}\left(c^{+}-c^{-}\right)\left(v-x^{1-v}\right) /(v-1)=\gamma+v\left(c^{+}-c^{-}\right) /(v-1) .
$$

So $E X_{1}>0$ if and only if $\gamma+v\left(c^{+}-c^{-}\right) /(v-1)>0$, and then the integral in (1.14) is finite if and only if $\alpha<v-1$. Thus for this process $E T_{r}^{1+\alpha}<\infty$, for an $\alpha \geqslant 0$, if and only if $v>1, \alpha<v-1$ and $\gamma+v\left(c^{+}-c^{-}\right) /(v-1)>0$. The conclusions remain the same if we also allow a Brownian component $(\sigma>0)$ in the process. 


\section{Proofs}

Our basic technique is to show that conditions such as (1.13)-(1.17) are equivalent to similar conditions for a certain random walk, and then appeal to results from [8]. Although at first sight this might seem mechanical or even routine, transferring probabilistic conditions such as (1.15) to the random walk setting is in fact quite delicate. An efficient way to proceed is by looking at the process at times at which it makes large jumps. We use the following recent result, taken from [4]. As previously mentioned, when $\Pi$ is not identically zero we assume (without loss of generality) that $c_{1}:=\Pi\left([-1,1]^{c}\right)=\bar{\Pi}^{+}(1)+\bar{\Pi}^{-}(1)>0$. Now put $\tau_{0}=0$, and for $n \geqslant 1$ write $\tau_{n}$ for the time at which $J_{n}$, the $n$th jump in $X$ whose absolute value exceeds 1, occurs. A random walk is then defined by

$$
\hat{S}:=\left(\hat{S}_{n}, n \geqslant 0\right), \quad \text { where } \hat{S}_{n}=X\left(\tau_{n}\right) .
$$

Of course $\left(\tau_{n}, n \geqslant 1\right)$ are the successive jump times of a Poisson process of rate $c_{1}$ which is independent of $\left(J_{n}, n \geqslant 1\right)$. We will write $\hat{Y}_{1}, \hat{Y}_{2}, \ldots$ for the steps in $\hat{S}$, so that, with $e_{n}:=\tau_{n}-\tau_{n-1}$, and $n \geqslant 1$, we have

$$
\hat{Y}_{n}=X\left(\tau_{n}\right)-X\left(\tau_{n-1}\right)=J_{n}+\tilde{X}\left(\tau_{n}\right)-\tilde{X}\left(\tau_{n-1}\right) \stackrel{D}{=} J_{n}+\tilde{X}\left(e_{n}\right),
$$

where $\tilde{X}$ is " $X$ with the jumps $J_{1}, J_{2}, \ldots$ removed". This process is also a Lévy process which can be written, in the notation of (1.2), as

$$
\tilde{X}_{t}=\gamma t+\sigma B_{t}+X_{t}^{(1)} \text {. }
$$

Furthermore $\tilde{X}$ is independent of $\left\{\left(J_{n}, \tau_{n}\right), n \geqslant 1\right\}$, and since it has no large jumps, it follows that $E\left(\mathrm{e}^{\theta \tilde{X}_{t}}\right)$ is finite for all real $\theta$. Thus the contribution of $\sum_{1}^{n}\left(\tilde{X}\left(\tau_{i}\right)-\tilde{X}\left(\tau_{i-1}\right)\right)$ to $\hat{S}_{n}$ can be easily estimated, and for our purposes $\hat{S}$ can be replaced by $S^{*}$, where $S_{n}^{*}=\sum_{1}^{n}\left(J_{r}+\tilde{\mu}\right)$, with $\tilde{\mu}=E \tilde{X}\left(\tau_{1}\right)$ (to see this, use Lemma 11 below). Next we introduce

$$
I_{n}:=\inf _{\tau_{n} \leqslant t<\tau_{n+1}} X_{t} \quad \text { and } \quad M_{n}=\sup _{\tau_{n} \leqslant t<\tau_{n+1}} X_{t},
$$

and also

$$
\tilde{i}_{n}=\inf _{0 \leqslant s<e_{n+1}}\left\{\tilde{X}\left(\tau_{n}+s\right)-\tilde{X}\left(\tau_{n}\right)\right\} \quad \text { and } \quad \tilde{m}_{n}=\sup _{0 \leqslant s<e_{n+1}}\left\{\tilde{X}\left(\tau_{n}+s\right)-\tilde{X}\left(\tau_{n}\right)\right\} .
$$

These quantities are independent of $\hat{S}_{n}$, and of course we have

$$
M_{n}=\hat{S}_{n}+\tilde{m}_{n}, \quad \text { and } \quad I_{n}=\hat{S}_{n}+\tilde{i}_{n} .
$$

However there is a different representation for the random variables $M_{n}$ and $I_{n}$ which turns out to be more useful:

Proposition 8 (Doney [4]). Using the above notation, we have

$$
M_{n}=S_{n}^{(+)}+\tilde{m}_{0} \quad \text { and } I_{n}=S_{n}^{(-)}+\tilde{i}_{0}, n \geqslant 0,
$$

where each of the processes $S^{(+)}=\left(S_{n}^{(+)}, n \geqslant 0\right)$ and $S^{(-)}=\left(S_{n}^{(-)}, n \geqslant 0\right)$ are random walks with the same distribution as $\hat{S}$. Moreover $S^{(+)}$and $\tilde{m}_{0}$ are independent, as are $S^{(-)}$and $\tilde{i}_{0}$.

For any random walk $S=\left(S_{n}, n \geqslant 0\right)\left(S_{0}=0\right)$ we will use the notation

$$
\Phi_{r}=\min \left(n \geqslant 0: S_{n}>r\right), \quad \Gamma_{r}=\max \left(n \geqslant 0: S_{n} \leqslant r\right), \quad r \geqslant 0
$$

(with $\Phi_{r}=\infty$ if $S_{n} \leqslant r$ for all $n \geqslant 0$ ), and the corresponding quantities for $\hat{S}, S^{(+)}$, and $S^{(-)}$will carry the appropriate superfix. It is obvious that Proposition 8 should enable us to compare the moments of $T_{r}$ and $\hat{\Phi}_{r}$, and the exact statement is:

Lemma 9. For any $r>0, E T_{r}^{1+\alpha}<\infty$ and $E \hat{\Phi}_{r}^{1+\alpha}<\infty$ are equivalent for $\alpha \geqslant 0$. For any $r \geqslant 0, E \Lambda_{r}^{\alpha}<\infty$ and $E \hat{\Gamma}_{r}^{\alpha}<\infty$ are equivalent for $\alpha>0$. In addition, $P\left(\Lambda_{r}<\infty\right)=1$ if and only if $P\left(\hat{\Gamma}_{r}<\infty\right)=1$. 
Proof. Since

$$
I_{n}=S_{n}^{(-)}+\tilde{i}_{0} \leqslant X_{t} \leqslant M_{n}=S_{n}^{(+)}+\tilde{m}_{0} \quad \text { for } \tau_{n} \leqslant t<\tau_{n+1},
$$

we deduce that for $n \geqslant 1$

$$
\left(\tau_{n}<T_{r} \leqslant \tau_{n+1}\right)=\left\{\tilde{m}_{0} \leqslant r, \Phi_{r-\tilde{m}_{0}}^{(+)}=n\right\},
$$

and hence, by Proposition 8 ,

$$
\sum_{n \geqslant 1} n^{\alpha} P\left(T_{r}>\tau_{n}\right) \asymp \int_{0}^{r} E\left(\hat{\Phi}_{r-y}^{1+\alpha}\right) P\left(\tilde{m}_{0} \in d y\right) \leqslant E\left(\hat{\Phi}_{r}^{1+\alpha}\right) .
$$

Also, for some $c>0$ and all large $r$,

$$
\sum_{n \geqslant 1} n^{\alpha} P\left(T_{r}>\tau_{n}\right) \geqslant c E\left(\hat{\Phi}_{r / 2}^{1+\alpha}\right) P\left(\tilde{m}_{0} \leqslant r / 2\right) \asymp E\left(\hat{\Phi}_{r / 2}^{1+\alpha}\right) .
$$

Next note that Theorem 2.1 of [8] implies that either $E \hat{\Phi}_{r}^{1+\alpha}<\infty$ for all $r \geqslant 0$, or $E \hat{\Phi}_{r}^{1+\alpha}=\infty$ for all $r \geqslant 0$. Thus to establish the first statement it suffices to show that

$$
E T_{r}^{1+\alpha}<\infty \quad \Leftrightarrow \quad \sum n^{\alpha} P\left(T_{r} \geqslant \tau_{n}\right)<\infty .
$$

Note that for any $c>0$,

$$
\left(T_{r} \geqslant n c\right) \subset\left\{\left(T_{r} \geqslant \tau_{n}\right) \cap\left(n c \geqslant \tau_{n}\right)\right\} \cup\left(\tau_{n}>n c\right),
$$

so that

$$
\sum n^{\alpha} P\left(T_{r} \geqslant n c\right) \leqslant \sum n^{\alpha} P\left(T_{r} \geqslant \tau_{n}\right)+\sum n^{\alpha} P\left(\tau_{n}>n c\right) .
$$

Since $\tau_{n}$ is $\operatorname{Gamma}\left(n, c_{1}\right)$, we see that the final term is finite whenever $c>1 / c_{1}$, and this gives one implication. For the other, note that

$$
\left(T_{r} \geqslant \tau_{n}\right) \subset\left(T_{r} \geqslant n c\right) \cup\left(\tau_{n}<n c\right),
$$

and choose $0<c<1 / c_{1}$; then $\sum P\left(\tau_{n}<n c\right)$ converges.

The results for $\Lambda_{r}$ depend on the observation that

$$
\left(\tau_{n}<\Lambda_{r} \leqslant \tau_{n+1}\right)=\left\{\Gamma_{r+\left|\tilde{i}_{0}\right|}^{(-)}=n\right\}, \quad n \geqslant 1,
$$

and are proved in a similar fashion.

To connect up with the other conditions in Theorem 1 we state a minor extension of Theorem 2.1 of [8]. If $Y$ is a typical step in a random walk $S$, we write $A_{+}(x)=\int_{0}^{x} P(Y>y) d y, x \geqslant 0$. We will also write $\Phi_{r}^{(\delta)}, \Gamma_{r}^{(\delta)}$ and $A_{+}^{(\delta)}(x)$ for $\Phi_{r}, \Gamma_{r}$ and $A_{+}(x)$ evaluated for the random walk $S^{(\delta)}:=\left(S_{n}-n \delta, n \geqslant 0\right)$.

Proposition 10. Let $S$ be any random walk with $\lim _{n \rightarrow \infty} S_{n}=\infty$ a.s., and write $\mu=E S_{1} \in(0, \infty)$ if $E\left|S_{1}\right|<\infty$, and $\mu=+\infty$ otherwise. Then for each fixed $\alpha \geqslant 0$, either the following three conditions all hold for each $0 \leqslant \delta<\mu$, or else they all fail for each such $\delta$ :

$$
\begin{aligned}
& \sum_{1}^{\infty} n^{\alpha-1} P\left(S_{n} \leqslant n \delta+r\right)<\infty \quad \text { for some (hence every) } r \geqslant 0 \\
& \int_{1}^{\infty}\left(\frac{x}{A_{+}^{(\delta)}(x)}\right)^{1+\alpha} P\left(Y^{-} \in d x\right)<\infty \\
& E\left(\Phi_{r}^{(\delta)}\right)^{1+\alpha}<\infty \quad \text { for some (hence every) } r \geqslant 0 \text { if } \alpha \geqslant 0 .
\end{aligned}
$$


Also equivalent to (2.8)-(2.10) for each $0 \leqslant \delta<\mu$, are

$$
\begin{aligned}
& E\left(\Gamma_{r}^{(\delta)}\right)^{\alpha}<\infty \text { for some (hence every) } r \geqslant 0, \text { if } \alpha>0, \\
& P\left(\Gamma_{r}^{(\delta)}<\infty\right)=1 \text { for some (hence every) } r \geqslant 0, \text { if } \alpha=0 .
\end{aligned}
$$

Proof. The restriction on $\delta$ guarantees that $\lim _{n \rightarrow \infty} S_{n}^{(\delta)}=\infty$ a.s., so for a fixed $\delta$ the above conditions are equivalent by virtue of Theorem 2.1 of [8]. (With reference to (2.9), note that since $\lim _{n \rightarrow \infty} S_{n}^{(\delta)}=\infty$ a.s., the first part of (2.5) of Theorem 2.1 of [8] is automatic, and Remark (ii) thereof shows that we can replace $A^{(\delta)}(y)$ by $A_{+}^{(\delta)}(y)$ in the second part.) However, when $\mu<\infty$, we have $0<A_{+}^{(\delta)}(\infty)<\infty$ for each $\delta$, and when $\mu=\infty$ we have $A_{+}^{(\delta)}(y) \sim A_{+}^{(0)}(y)$ as $y \rightarrow \infty$ for each $\delta$, so it is easy to see that (2.9) either holds for all allowable $\delta$, or for none.

We now prove several lemmas, preparing for the proof of Theorem 1.

Lemma 11. Let $S, \tilde{S}$ be random walks with typical steps $Y, \tilde{Y}$ such that

$$
\tilde{Y} \stackrel{D}{=} Y+W
$$

where $Y$ and $W$ are independent, $E W=0$ and $E \mathrm{e}^{\theta W}$ is finite for $0 \leqslant|\theta| \leqslant \theta_{0}$, some $\theta_{0}>0$. Then $\lim _{n \rightarrow \infty} S_{n}=$ $\infty$ a.s. if and only if $\lim _{n \rightarrow \infty} \tilde{S}_{n}=\infty$ a.s., and for $\alpha \geqslant 0, r \geqslant 0, \sum_{1}^{\infty} n^{\alpha-1} P\left(S_{n} \leqslant r\right)<\infty$ if and only if $\sum_{1}^{\infty} n^{\alpha-1} P\left(\tilde{S}_{n} \leqslant r\right)<\infty$.

Proof. Since $\lim _{n \rightarrow \infty} S_{n}=\infty$ a.s. occurs if and only if $n^{-1} S_{n} \stackrel{\text { a.s. }}{\rightarrow} \mu \in(0, \infty]$, (see [6]) and $n^{-1}\left(\tilde{S}_{n}-S_{n}\right) \stackrel{\text { a.s. }}{\rightarrow} 0$ by the strong law, the first statement is immediate. For the second, note first that

$$
P\left(\tilde{S}_{n} \leqslant r\right) \geqslant P\left(S_{n} \leqslant r\right) P\left(\sum_{1}^{n} W_{i} \leqslant 0\right) \sim \frac{1}{2} P\left(S_{n} \leqslant r\right) .
$$

On the other hand, choosing $\delta \in(0, \mu)$ and using a standard exponential bound for sums of i.i.d. rvs with a finite moment generating function,

$$
\begin{aligned}
P\left(\tilde{S}_{n} \leqslant r\right) & =P\left(S_{n}^{(\delta)}+\sum_{1}^{n}\left(W_{i}+\delta\right) \leqslant r\right) \leqslant P\left(S_{n}^{(\delta)} \leqslant r\right)+P\left(\sum_{1}^{n} W_{i} \leqslant-n \delta\right) \\
& =P\left(S_{n}^{(\delta)} \leqslant r\right)+\mathrm{O}\left(\mathrm{e}^{-n c}\right) \text { as } n \rightarrow \infty,
\end{aligned}
$$

where $c>0$, and the result follows from Proposition 10 .

Lemma 12. Let $S$ be any random walk, $M$ an independent Poisson process of parameter $\lambda, a \in \mathbb{R}$, and $\tilde{Z}$ a Lévy process which is independent of $S$ and $M$, has $E \tilde{Z}_{1}=0$, and has $E \mathrm{e}^{\theta} \tilde{Z}_{1}$ finite for $0 \leqslant|\theta| \leqslant \theta_{0}$, for some $\theta_{0}>0$. Define $Z_{t}=S_{M_{t}}+a\left(M_{t}-\lambda t\right)+\tilde{Z}_{t}$. Then: (i) for $\alpha \geqslant 0, r \geqslant 0$,

$$
\int_{1}^{\infty} t^{\alpha-1} P\left(Z_{t} \leqslant r\right) d t \asymp \sum_{1}^{\infty} n^{\alpha-1} P\left(S_{n} \leqslant r\right),
$$

in the sense that if one side is finite, so is the other, and if one side is finite for an $r \geqslant 0$, then the ratio of the two sides is bounded away from 0 and infinity as $r \rightarrow \infty$; (ii) $\lim _{t \rightarrow \infty} Z_{t}=\infty$ a.s. if and only if $\lim _{n \rightarrow \infty} S_{n}=\infty$ a.s. 
Proof. (i) By the central limit theorem, $\lim _{t \rightarrow \infty} P\left(\tilde{Z}_{t} \leqslant 0\right)=1 / 2$, so we have, as $t \rightarrow \infty$,

$$
P\left(Z_{t} \leqslant r\right) \geqslant P\left(\tilde{Z}_{t} \leqslant 0\right) P\left(S_{M_{t}}+a\left(M_{t}-\lambda t\right) \leqslant r\right) \sim P\left(S_{M_{t}}+a\left(M_{t}-\lambda t\right) \leqslant r\right) / 2 .
$$

Also, if $a \geqslant 0$,

$$
P\left(S_{M_{t}}+a\left(M_{t}-\lambda t\right) \leqslant r\right) \geqslant P\left(S_{M_{t}} \leqslant r, M_{t}-\lambda t \leqslant 0\right)=\sum_{0 \leqslant n \leqslant \lambda t} \mathrm{e}^{-\lambda t} \frac{(\lambda t)^{n}}{n !} P\left(S_{n} \leqslant r\right) .
$$

Thus

$$
\begin{aligned}
\int_{1}^{\infty} t^{\alpha-1} P\left(S_{M_{t}}+a\left(M_{t}-\lambda t\right) \leqslant r\right) d t & \geqslant \sum_{n \geqslant 0} \frac{\lambda^{n}}{n !} P\left(S_{n} \leqslant r\right) \int_{n / \lambda}^{\infty} t^{n+\alpha-1} \mathrm{e}^{-\lambda t} d t \\
& =\lambda^{-\alpha} \sum_{n \geqslant 0} \frac{1}{n !} P\left(S_{n} \leqslant r\right) \int_{n}^{\infty} s^{n+\alpha-1} \mathrm{e}^{-s} d s \\
& =\lambda^{-\alpha} \sum_{n \geqslant 0} \frac{\Gamma(n+\alpha)}{n !} P\left(S_{n} \leqslant r\right) P\left(\Theta_{n+\alpha} \geqslant n\right),
\end{aligned}
$$

where $\Theta_{n+\alpha}$ denotes a random variable having a Gamma distribution with parameters $n+\alpha$ and 1 . By the central limit theorem again, we have $\liminf _{n \rightarrow \infty} P\left(\Theta_{n+\alpha} \geqslant n\right) \geqslant 1 / 2$, so, using Stirling's formula, the summand in (2.14) is seen to be bounded below by a constant times $n^{\alpha-1} P\left(S_{n} \leqslant r\right)$. Thus, assuming that $a \geqslant 0$, the finiteness of the left-hand side of (2.12) implies the finiteness of the right-hand side. If $a<0$, an analogous argument with $M_{t}-\lambda t>0$ in (2.13) gives (2.14) with $P\left(\lambda<\Theta_{n+\alpha} \leqslant n\right)$ in place of $P\left(\Theta_{n+\alpha} \geqslant n\right)$, and we reach the same conclusion in a similar way.

For the reverse implication, assume that the series in (2.12) converges, so that $\lim _{n \rightarrow \infty} S_{n}=\infty$ a.s., then choose $0<\delta<\mu=E S_{1}$, where the final equality is in the sense used in Proposition 10, and again write $S^{(\delta)}=\left(S_{n}-n \delta\right.$, $n \geqslant 0)$. Then

$$
\begin{aligned}
P\left(Z_{t} \leqslant r\right) & =P\left(S_{M_{t}}^{(\delta)}+\delta M_{t}+a\left(M_{t}-\lambda t\right)+\tilde{Z}_{t} \leqslant r\right) \\
& \leqslant P\left(S_{M_{t}}^{(\delta)} \leqslant r\right)+P\left((1+a / \delta)\left(M_{t}-\lambda t\right)+\delta^{-1} \tilde{Z}_{t} \leqslant-\lambda t\right) \\
& =P\left(S_{M_{t}}^{(\delta)} \leqslant r\right)+\mathrm{O}\left(\mathrm{e}^{-c t}\right) \quad \text { uniformly in } r \geqslant 0, \text { as } t \rightarrow \infty,
\end{aligned}
$$

where we have again used a standard exponential bound, applicable because $(1+a / \delta)\left(M_{1}-\lambda\right)+\delta^{-1} \tilde{Z}_{1}$ has zero mean and finite moment generating function on $\left(-\theta_{0}, \theta_{0}\right)$. However, by Proposition 10 we have $\sum_{1}^{\infty} n^{\alpha-1} P\left(S_{n}^{(\delta)} \leqslant\right.$ $r)<\infty$, and we conclude by the calculation

$$
\int_{1}^{\infty} t^{\alpha-1} P\left(S_{M_{t}}^{(\delta)} \leqslant r\right) d t \leqslant \int_{0}^{\infty} t^{\alpha-1} \sum_{n \geqslant 0} \mathrm{e}^{-\lambda t} \frac{(\lambda t)^{n}}{n !} P\left(S_{n}^{(\delta)} \leqslant r\right) d t=\lambda^{-\alpha} \sum_{n \geqslant 0} \frac{\Gamma(n+\alpha)}{n !} P\left(S_{n}^{(\delta)} \leqslant r\right) .
$$

(ii) The second part follows since the left-hand side of (2.12) is finite for $\alpha=0$ if and only if $\lim _{t \rightarrow \infty} Z_{t}=\infty$ a.s. by (1.10), and the right-hand side of (2.12) is finite for $\alpha=0$ if and only if $\lim _{n \rightarrow \infty} S_{n}=\infty$ a.s. by Spitzer's theorem (or Theorem 2.1 of [8]).

Lemma 13. (i) If $A(x)>0$ for all large $x$ and (1.14) holds then $\lim _{t \rightarrow \infty} X_{t}=\infty$ a.s. 
(ii) Whenever $\lim _{t \rightarrow \infty} X_{t}=\infty$ a.s. it follows that $\lim _{x \rightarrow \infty} A(x)$ exists and is positive (possibly $\left.+\infty\right)$, thus $A(x)$ is positive for all large enough $x$, and

$$
A(x) \asymp \int_{1}^{x} \bar{\Pi}^{+}(y) d y \quad \text { as } x \rightarrow \infty .
$$

Proof. (i) Suppose $A(x)>0$ for all $x \geqslant x_{0}$ and (1.14) holds. Then

$$
\int_{\left(x_{0}, \infty\right)}\left(\frac{x}{A(x)}\right) \Pi^{\#}(d x)<\infty .
$$

If $\int_{1}^{\infty} \bar{\Pi}^{+}(x) d x=\infty$, then from (1.7)

$$
A(x) \leqslant \gamma+\bar{\Pi}^{+}(1)+\int_{1}^{x} \bar{\Pi}^{+}(y) d y \sim \int_{1}^{x} \bar{\Pi}^{+}(y) d y,
$$

so by (2.16) (see (1.8) for $J_{-}$)

$$
J_{-} \leqslant \int_{(1, \infty)} \frac{x \Pi^{\#}(d x)}{\int_{1}^{x} \bar{\Pi}^{+}(y) d y}<\infty
$$

If $\int_{1}^{\infty} \bar{\Pi}^{+}(x) d x<\infty$ then (1.18) gives $0<E X_{1} \leqslant E\left|X_{1}\right|<\infty$. Thus $\lim _{t \rightarrow \infty} X_{t}=\infty$ a.s., by (1.9).

(ii) Assuming that $\lim _{t \rightarrow \infty} X_{t}=\infty$ a.s. and $\int_{1}^{\infty} \bar{\Pi}^{+}(x) d x=\infty$, then, since $x^{-1} \int_{1}^{x} \bar{\Pi}^{+}(y) d y$ is nonincreasing, it follows from the convergence of $J_{-}$in (1.9) that $\int_{1}^{x} \bar{\Pi}^{-}(y) d y=\mathrm{o}\left(\int_{1}^{x} \bar{\Pi}^{+}(y) d y\right)$ as $x \rightarrow \infty$. So from (1.7), $A(x) \sim \int_{1}^{x} \bar{\Pi}^{+}(y) d y$ and $A(\infty)=+\infty>0$. This gives (2.15).

If $\lim _{t \rightarrow \infty} X_{t}=\infty$ a.s. and $\int_{1}^{\infty} \bar{\Pi}^{+}(x) d x<\infty$, then $0<E X_{1} \leqslant E\left|X_{1}\right|<\infty$ by (1.9), and $A(\infty)=E X_{1}$, so $A(x)>0$ for large $x$.

Proof of Theorem 1. Fix $\alpha \geqslant 0$ throughout. Assume (1.13) for some $r>0$, so that $\lim _{t \rightarrow \infty} X_{t}=\infty$ a.s. by (1.10). Using the notation of Proposition 8 and Lemma 9, from the bound

$$
S_{N_{t}}^{(-)}+\tilde{i}_{0} \leqslant X_{t} \leqslant S_{N_{t}}^{(+)}+\tilde{m}_{0}
$$

where $N_{t}=\max \left\{n: \tau_{n} \leqslant t\right\}$, we see that $\lim _{t \rightarrow \infty} S_{N_{t}}^{(+)}=\infty$ a.s. Part (ii) of Lemma 12 (with $a=0$ and $\tilde{Z}_{t} \equiv 0$ ) then gives $\lim _{n \rightarrow \infty} S_{n}^{(+)}=\infty$ a.s., so $\lim _{n \rightarrow \infty} \hat{S}_{n}=\infty$ a.s. By (2.1) and (2.2) we can write, with $\tilde{\mu}=E \tilde{X}\left(\tau_{1}\right)=$ $E \tau_{1} E \tilde{X}_{1}=\gamma / c_{1}$,

$$
\hat{S}_{n}=\sum_{i=1}^{n}\left(J_{i}+\tilde{\mu}\right)+\sum_{i=1}^{n}\left(\tilde{X}\left(\tau_{i}\right)-\tilde{X}\left(\tau_{i-1}\right)-\tilde{\mu}\right)=S_{n}^{*}+\sum_{i=1}^{n} W_{i}, \quad \text { say. }
$$

Then Lemma 11 gives $\lim _{n \rightarrow \infty} S_{n}^{*}=\infty$ a.s. Now $N_{t}$ is the same as that occurring in (1.11), and we can rewrite (1.2) in the form

$$
X_{t}=\gamma t+\sigma B_{t}+X_{t}^{(1)}+\sum_{i=1}^{N_{t}} J_{i}=S_{N_{t}}^{*}+\frac{\gamma}{c_{1}}\left(c_{1} t-N_{t}\right)+\sigma B_{t}+X_{t}^{(1)}=S_{N_{t}}^{*}+\frac{\gamma}{c_{1}}\left(c_{1} t-N_{t}\right)+\bar{X}_{t},
$$

where $\bar{X}$ is independent of $S^{*}$ and $N$, and has $E \bar{X}_{1}=0$ and $E \mathrm{e}^{\theta \bar{X}_{1}}$ finite for all real $\theta$. Thus we have precisely the setup of Lemma 12, and we deduce from it that $\sum_{1}^{\infty} n^{\alpha-1} P\left(S_{n}^{*} \leqslant r\right)<\infty$, or equivalently, by Lemma 11 , 
$\sum_{1}^{\infty} n^{\alpha-1} P\left(\hat{S}_{n} \leqslant r\right)<\infty$. By Lemma 9 and Proposition 10 applied to $\hat{S}$ with $\delta=0$, this is equivalent to (1.15) and the two conditions in (1.16), and also to

$$
\int_{0}^{\infty}\left(\frac{x}{\int_{0}^{x} P\left(J_{1}+\tilde{\mu}>y\right) d y}\right)^{1+\alpha} P\left(\left(J_{1}+\tilde{\mu}\right)^{-} \in d x\right)<\infty .
$$

Thus (1.13), (1.15), and the two conditions in (1.16), and (2.20), are equivalent.

Assuming any of these, we have $\lim _{t \rightarrow \infty} X_{t}=\infty$ a.s., so $A(x)>0$ for all large $x$, in view of part (ii) of Lemma 13. Also, (1.14) follows from (2.20). Indeed either $\int_{0}^{\infty} P\left(J_{1}+\tilde{\mu}>x\right) d x<\infty$, in which case $A(\infty)=$ $E X_{1} \in(0, \infty)$ and both (2.20) and (1.14) reduce to $\int_{1}^{\infty} x^{1+\alpha} \Pi^{\#}(d x)<\infty$, or $\int_{0}^{\infty} P\left(J_{1}+\tilde{\mu}>x\right) d x=\infty$, in which case $\int_{0}^{x} P\left(J_{1}+\tilde{\mu}>y\right) d y \asymp \int_{1}^{x} \bar{\Pi}^{+}(y) d y$ as $x \rightarrow \infty$, and the equivalence follows from Lemma 13.

Conversely, if $A(x)>0$ for all large $x$, and (1.14) holds, then Lemma 13 gives $\lim _{t \rightarrow \infty} X_{t}=\infty$ a.s. (and hence $\lim _{n \rightarrow \infty} \hat{S}_{n}=\infty$ a.s.), and also enables us to check that (2.20) holds, whence the other statements, except (1.17), follow.

Before dealing with (1.17), we need some notation.

Definition 14. Say that a real-valued function $f$ is in $\mathcal{C}_{\alpha}$, where $\alpha \geqslant 0$, if, with $k=[\alpha]$, there exist real constants $c_{1}, c_{2}, \ldots, c_{k}$ such that

$$
f(x)=\sum_{1}^{k} c_{j} x^{j}+\varepsilon(x)
$$

where, if $\alpha$ is an integer, $\varepsilon(x)=\mathrm{o}\left(x^{k}\right)$ as $x \downarrow 0$, and otherwise

$$
\int_{0}^{1}|\varepsilon(x)| x^{-\alpha-1} d x<\infty .
$$

Note that if $f \in \mathcal{C}_{\alpha}$ then for any integer $m \geqslant 1, x^{m} f(x) \in \mathcal{C}_{\alpha}$, and hence $f^{m}(x) \in \mathcal{C}_{\alpha}$. This leads to:

Definition 15. Say that a power series of the form $P(s)=\sum_{1}^{\infty} a_{n} s^{n}$ with real coefficients is in class $\mathcal{P}$ if (i) $a_{1} \neq 0$, (ii) it has a positive radius of convergence and (iii) on some nondegenerate interval $(0, \delta)$ it has a well-defined inverse $P^{-1}$ which is another power series which satisfies (i) and (ii).

Remark. (vi) The function $P(x)=-\log (1-x)$, with inverse $P^{-1}(x)=1-\exp (-x)$, has a power series expansion which is in $\mathcal{P}$.

Lemma 16. A nonnegative random variable $Y$ has a finite moment of order $\alpha>0$ if and only if, with $\phi(q)=$ $E\left(\mathrm{e}^{-q Y}\right)$,

$$
P(1-\phi(\cdot)) \in \mathcal{C}_{\alpha}, \quad \text { for every } P \in \mathcal{P} .
$$

Proof. From [2] (the result is also quoted as Theorem 8.18, p. 335, of [3]) we know that $E Y^{\alpha}<\infty$ is equivalent to $1-\phi(\cdot) \in \mathcal{C}_{\alpha}$. But if this happens then for $k=1,2, \ldots$, each of $(1-\phi(\cdot))^{k} \in \mathcal{C}_{\alpha}$, and hence so also does $\sum_{1}^{k} a_{n}(1-\phi(\cdot))^{n}$ when $P \in \mathcal{P}$. Since $\left|\sum_{k+1}^{\infty} a_{n} s^{n}\right| \leqslant c|s|^{k+1}$ for all sufficiently small $|s|$, we see that $P(1-\phi(\cdot)) \in$ $\mathcal{C}_{\alpha}$. On the other hand, if $\psi(\cdot):=P(1-\phi(\cdot)) \in \mathcal{C}_{\alpha}$, then since $P^{-1} \in \mathcal{P}$, we have $1-\phi(\cdot)=P^{-1} \psi(\cdot) \in \mathcal{C}_{\alpha}$, and hence $E Y^{\alpha}<\infty$. 
Lemma 17. Suppose the functions $P_{1}$ and $P_{2}$ are each in $\mathcal{P}$, and $B(s)=\sum_{1}^{\infty} b_{n} s^{n}$ is another power series satisfying conditions (i) and (ii) in Definition 15. Suppose that $Y_{1}$ and $Y_{2}$ are nonnegative random variables, and $\phi_{i}(q)=E\left(\mathrm{e}^{-q Y_{i}}\right), i=1,2$, satisfy

$$
P_{1}\left(1-\phi_{1}(q)\right)=B(q)\left(1+P_{2}\left(1-\phi_{2}(q)\right)\right), \quad q \geqslant 0 .
$$

Then for $\alpha>0, E Y_{1}^{1+\alpha}<\infty \Leftrightarrow E Y_{2}^{\alpha}<\infty$.

Proof. Assume $E Y_{2}^{\alpha}<\infty$. Then by Lemma 16, $P_{2}\left(1-\phi_{2}(\cdot)\right) \in \mathcal{C}_{\alpha}$, and it follows easily that

$$
P_{1}\left(1-\phi_{1}(\cdot)\right)=B(\cdot)\left(1+P_{2}\left(1-\phi_{2}(\cdot)\right)\right) \in \mathcal{C}_{1+\alpha},
$$

so $E Y_{1}^{1+\alpha}<\infty$ by Lemma 16 again. For the converse, assume $E Y_{1}^{1+\alpha}<\infty$, so that $P_{1}\left(1-\phi_{1}(\cdot)\right) \in \mathcal{C}_{1+\alpha}$, and hence can be written in the form

$$
P_{1}\left(1-\phi_{1}(q)\right)=\sum_{1}^{k+1} c_{j} q^{j}+\varepsilon(q) .
$$

Then from (2.23)

$$
1=\lim _{q \downarrow 0} \frac{P_{1}\left(1-\phi_{1}(q)\right)}{B(q)}=\frac{c_{1}}{b_{1}},
$$

and again it is easy to see that

$$
P_{2}\left(1-\phi_{2}(\cdot)\right)=\frac{P_{1}\left(1-\phi_{1}(\cdot)\right)}{B(\cdot)}-1 \in \mathcal{C}_{\alpha},
$$

and the result follows.

To prove (1.17) equivalent with the other conditions in Theorem 1, let us first note that from the Weiner-Hopf factorisation (Bertoin [1, p. 166]), we have

$$
(a q)^{-1} \kappa(q)=-(a q)^{-1} \log E\left(\mathrm{e}^{-q L^{-1}(1)}\right)=\exp \left\{\int_{0}^{\infty} t^{-1}\left(\mathrm{e}^{-q t}-\mathrm{e}^{-t}\right) P\left(X_{t} \leqslant 0\right) d t\right\},
$$

for $q>0$, for some constant $a>0$. Letting $q \downarrow 0$ we see that

$$
E L^{-1}(1)<\infty \Leftrightarrow \int_{0}^{\infty} t^{-1}\left(1-\mathrm{e}^{-t}\right) P\left(X_{t} \leqslant 0\right) d t<\infty \Leftrightarrow \int_{1}^{\infty} t^{-1} P\left(X_{t} \leqslant 0\right) d t<\infty,
$$

or, equivalently, by (1.10), $\lim _{t \rightarrow \infty} X_{t}=\infty$ a.s. Thus when any of (1.13)-(1.17) hold, we have

$$
K:=\int_{1}^{\infty} t^{-1} P\left(X_{t} \leqslant 0\right) d t<\infty
$$

so that we can define a probability density function on $t \geqslant 0$ by

$$
f(t)=\frac{P\left(X_{t} \leqslant 0\right) \mathbf{1}_{\{t \geqslant 1\}}}{t K} .
$$

Next, write the integral in (2.24) as

$$
-K \int_{0}^{\infty}\left(1-\mathrm{e}^{-q t}\right) f(t) d t+\int_{1}^{\infty} t^{-1}\left(1-\mathrm{e}^{-t}\right) P\left(X_{t} \leqslant 0\right) d t+\int_{0}^{1} t^{-1}\left(\mathrm{e}^{-q t}-\mathrm{e}^{-t}\right) P\left(X_{t} \leqslant 0\right) d t,
$$


so that

$$
-\log \phi_{1}(q):=\kappa(q)=B(q) \exp \left(-K\left(1-\phi_{2}(q)\right)\right),
$$

where $\phi_{2}(q)=\int_{0}^{\infty} \mathrm{e}^{-q t} f(t) d t$,

$$
B(q)=\tilde{a} q \exp \left\{\int_{0}^{1} t^{-1}\left(\mathrm{e}^{-q t}-\mathrm{e}^{-t}\right) P\left(X_{t} \leqslant 0\right) d t\right\}
$$

and

$$
\tilde{a}=a \exp \left\{\int_{1}^{\infty} t^{-1}\left(1-\mathrm{e}^{-t}\right) P\left(X_{t} \leqslant 0\right) d t\right\}
$$

Thus, with $P_{1}(x)=-\log (1-x)$ and $P_{2}(x)=-1+\exp (-K x)$ we have the setup of Lemma 17 with $L^{-1}(1)=Y_{1}$, and $Y_{2}$ having $f(\cdot)$ as its probability density function. Since it is easily seen that each of $P_{1}, P_{2}$ and $B$ satisfy the assumptions in that lemma, and $E\left(Y_{2}^{\alpha}\right)=K^{-1} \int_{1}^{\infty} t^{\alpha-1} P\left(X_{t} \leqslant 0\right) d t$, the result follows.

Proof of Theorem 2. (i) Keep $0<\alpha<1$ and $r>0$. Integrate by parts in (1.20) to get

$$
\int_{0}^{\infty}\left(1-\mathrm{e}^{-q t}\right) P\left(T_{r} \in d t\right)=\kappa(q) V^{q}(r)
$$

Dividing by $q^{\alpha+1}$ and integrating each side gives

$$
c_{\alpha} E T_{r}^{\alpha}=\int_{0}^{\infty} t^{\alpha}\left(\int_{0}^{\infty} q^{-\alpha-1}\left(1-\mathrm{e}^{-q}\right) d q\right) P\left(T_{r} \in d t\right)=\int_{0}^{\infty} q^{-\alpha-1} \kappa(q) V^{q}(r) d q,
$$

in the sense that if one side is finite, so is the other, and they are equal, with $c_{\alpha}=\Gamma(1-\alpha) / \alpha$ as the value of the inner integral. This establishes (1.22).

Now by $(2.25), \kappa(q) V^{q}(r) \leqslant 1$ for all $q>0, r \geqslant 0$, so, since $\alpha>0$, the integral $\int_{1}^{\infty} q^{-\alpha-1} \kappa(q) V^{q}(r) d q$ is finite for all $r \geqslant 0$. Thus $E T_{r}^{\alpha}<\infty$ if and only if $q^{-\alpha-1} \kappa(q) V^{q}(r) \in L(0,1)$. But since, for each $r>0$, as $q \downarrow 0$,

$$
V^{q}(r) \uparrow V(r):=\int_{0}^{\infty} P\left(H_{t} \leqslant r\right) d t \in(0, \infty),
$$

we see that $E T_{r}^{\alpha}<\infty$ if and only if $q^{-\alpha-1} \kappa(q) \in L(0,1)$. From (2.24) we then see that (1.21) holds.

(ii) Now assume $\lim _{t \rightarrow \infty} X_{t}=+\infty$ a.s., or, equivalently, $E T_{r}<\infty$. From (1.20) we get

$$
E T_{r}=a^{\prime} V(r)
$$

where $a^{\prime}=\lim _{q \rightarrow 0} \kappa(q) / q \in(0, \infty)$. By Bertoin [1, p. 74],

$$
V(r)=\int_{0}^{\infty} P\left(H_{t} \leqslant r\right) d t \asymp \frac{r}{\int_{0}^{r} \bar{\Pi}_{H}(y) d y},
$$

where $\Pi_{H}(y)$ is the Lévy measure of the subordinator $H$. Using Proposition 3.3 of Vigon [12], we can write

$$
\bar{\Pi}_{H}(r)=\int_{0}^{\infty} V^{\#}(d y) \bar{\Pi}_{X}^{+}(r+y) \leqslant V^{\#}([0, \infty)) \bar{\Pi}_{X}^{+}(r)=c^{\prime} \bar{\Pi}_{X}^{+}(r),
$$


where $V^{\#}([0, r])=\int_{0}^{\infty} P\left(H_{t}^{\#} \leqslant r\right) d t$. Note that $c^{\prime}=V^{\#}([0, \infty))<\infty$ since $X$ drifts to $+\infty$ a.s. In the other direction, take $c>0$ such that $V^{\#}([0, c])=c^{\prime \prime}>0$, as is possible since $X$ is not a subordinator, and get

$$
\bar{\Pi}_{H}(r) \geqslant \int_{0}^{c} V^{\#}(d y) \bar{\Pi}_{X}^{+}(r+c) \geqslant c^{\prime \prime} \bar{\Pi}_{X}^{+}(r+c) .
$$

From these we easily see that $\int_{0}^{r} \bar{\Pi}_{H}(y) d y \asymp \int_{1}^{r} \bar{\Pi}_{X}^{+}(y) d y$ and thus, since $A(r) \asymp \int_{0}^{r} \bar{\Pi}_{X}^{+}(y) d y$, we have $\int_{0}^{r} \bar{\Pi}_{H}(y) d y \asymp A(r)$.

Proof of Theorem 3. (i) Under the hypotheses of Theorem 3, we have $\lim _{t \rightarrow \infty} X_{t}=\infty$ a.s. and $A(x)>0$ for all large $x$. As in the proof of Theorem 1, again we have $\lim _{n \rightarrow \infty} \hat{S}_{n}=\infty$ a.s. and $\lim _{n \rightarrow \infty} S_{n}^{*}=\infty$ a.s. So Theorem 2.2 of [8] gives, as $r \rightarrow \infty$,

$$
\sum_{1}^{\infty} n^{\alpha-1} P\left(\hat{S}_{n} \leqslant r\right) \asymp\left(\frac{r}{\hat{A}(r)}\right)^{\alpha} \text { and } \sum_{1}^{\infty} n^{\alpha-1} P\left(S_{n}^{*} \leqslant r\right) \asymp\left(\frac{r}{A^{*}(r)}\right)^{\alpha},
$$

when $\alpha>0$, or with logarithmic terms on the right when $\alpha=0$, provided the series converge; and this is the case when the integral in (1.24) converges, by Theorem 1 and its proof (even for $\alpha=0$ ). However, the proofs of Proposition 10 and Lemma 11 show that we have $\hat{A}(r) \sim \hat{A}_{+}(r) \sim A_{+}^{*}(r) \sim A^{*}(r) \sim A(r)$ as $r \rightarrow \infty$, so (1.24) and the outer relation in (1.27) follow from (2.12) of Lemma 12.

To get (1.25), use the argument in Lemma 9 to see that for suitable $c$ and $\alpha>0$

$$
E T_{r}^{\alpha}=\alpha \int_{0}^{\infty} t^{\alpha-1} P\left(T_{r}>t\right) d t \asymp \sum_{1}^{\infty} n^{\alpha-1} P\left(T_{r}>n c\right) \asymp \sum_{1}^{\infty} n^{\alpha-1} P\left(T_{r}>\tau_{n}\right) \asymp E\left(\hat{\Phi}_{\left(r-\tilde{m}_{0}\right)^{+}}^{\alpha}\right) .
$$

Since Theorem 2.2 of [8] also gives

$$
E \hat{\Phi}_{r}^{\alpha} \asymp\left(\frac{r}{\hat{A}(r)}\right)^{\alpha} \asymp\left(\frac{r}{\hat{A}_{+}(r)}\right)^{\alpha} \text { as } r \rightarrow \infty,
$$

we see, by dominated convergence and the fact that $E \tilde{m}_{0}^{\alpha}<\infty$, that

$$
E \hat{\Phi}_{\left(r-\tilde{m}_{0}\right)^{+}}^{\alpha} \asymp\left(\frac{r}{\hat{A}_{+}(r)}\right)^{\alpha} \asymp\left(\frac{r}{A(r)}\right)^{\alpha} \quad \text { as } r \rightarrow \infty .
$$

The proof of (1.26) follows the same lines.

(ii) For the remainder of (1.27): the inner integral is clearly smaller than the left-hand integral. In the other direction, use (2.18) to write

$$
\sup _{0 \leqslant s \leqslant t} X_{s} \leqslant \sup _{0 \leqslant s \leqslant t} S_{N_{s}}^{(+)}+\tilde{m}_{0} .
$$

Let $Y_{i}$ be the steps in $S_{n}^{(+)}$. Now $\lim _{n \rightarrow \infty} S_{n}^{(+)}=\infty$ a.s. so $\sum_{1}^{n} Y_{i}^{-}=\mathrm{o}\left(\sum_{1}^{n} Y_{i}^{+}\right)$a.s., if $E\left|Y_{1}\right|=\infty$ (see Pruitt [9]), while $\sum_{1}^{n} Y_{i}^{-} \sim\left(E Y_{1}^{+} / E Y_{1}-1\right) \sum_{1}^{n} Y_{i}$ a.s., if $0<E Y_{1} \leqslant E\left|Y_{1}\right|<\infty$. Thus $\sum_{1}^{n} Y_{i}^{+}=\mathrm{O}\left(\sum_{1}^{n} Y_{i}\right)$ a.s., as $n \rightarrow \infty$. It follows that

$$
\begin{aligned}
\sup _{0 \leqslant s \leqslant t} S_{N_{s}}^{(+)} & =\sup _{0 \leqslant s \leqslant t}\left(\sum_{i=1}^{N_{s}} Y_{i}\right) \leqslant \sup _{0 \leqslant s \leqslant t}\left(\sum_{i=1}^{N_{s}} Y_{i}^{+}\right) \\
& =\sum_{i=1}^{N_{t}} Y_{i}^{+}=\mathrm{O}\left(\sum_{1}^{N_{t}} Y_{i}\right)=\mathrm{O}\left(S_{N_{t}}^{(+)}\right) \quad \text { a.s., as } t \rightarrow \infty .
\end{aligned}
$$


Since $S_{N_{t}}^{(+)}$and $S_{N_{t}}^{(-)}$have the same distribution, (2.18) shows that we can replace $\sup _{0 \leqslant s \leqslant t} X_{s}$ by $X_{t}$ for the purposes of proving (1.27). Then the result follows from the already-established outer relation in (1.27).

Proof of Theorem 4. The proofs that the quantities in (1.28) and (1.29) have finite moments of order $\alpha$ are based on the facts that

$$
\left(\tau_{n}<T(-r) \leqslant \tau_{n+1}\right)=\left\{\left|\tilde{i}_{0}\right| \leqslant r, \Phi^{(+)}\left(-\left(r+\tilde{i}_{0}\right)\right)=n\right\},
$$

and

$$
\left(\tau_{n}<T_{\min } \leqslant \tau_{n+1}\right)=\left\{I_{\infty}<\tilde{i}_{0}, \Phi_{\min }^{(-)}=n\right\},
$$

where $\Phi^{(+)}(-r)$ and $\Phi_{\min }^{(-)}$have their obvious meanings. We omit the details.

For (1.30), we have $O_{r} \leqslant \Lambda_{r}$, so each of the conditions in (1.16) implies that (1.30) has a finite moment of the corresponding order $\alpha$; conversely,

$$
O_{r} \geqslant O_{0}=\int_{0}^{\infty} \mathbf{1}_{\left\{X_{s}<0\right\}} d s=\lim _{t \rightarrow \infty} \int_{0}^{t} \mathbf{1}_{\left\{X_{s}<0\right\}} d s .
$$

However the Sparre-Andersen Identity (Bertoin [1, p. 170]) states that

$$
\int_{0}^{t} \mathbf{1}_{\left\{X_{s}<0\right\}} d s \stackrel{D}{=} T_{\min }(t):=\inf \left\{s: X_{s} \leqslant I_{t} \text { or } X_{s-} \leqslant I_{t}\right\} \quad \text { where } I_{t}=\inf _{0 \leqslant s<t} X_{s},
$$

and we conclude that $O_{0} \stackrel{D}{=} T_{\min }$. Thus if $O_{r}$ has a finite moment of order $\alpha$, then so does $T_{\min }$.

Proof of Theorem 5. We start by observing that a minor variation of the proof of Theorem 1 shows that (1.31) is equivalent to the condition (4.14) of [8] evaluated either for $\hat{S}$ or $S^{*}$. Next, note that

$$
I_{\infty}=\tilde{i}_{0}+\min _{n \geqslant 0} S_{n}^{(-)} \stackrel{D}{=} \tilde{i}_{0}+\min _{n \geqslant 0} \hat{S}_{n}
$$

where the two terms are independent. Since $\left|\tilde{i}_{0}\right|$ has finite moments of all orders, it is clear that $E\left(\left|I_{\infty}\right|^{\alpha}\right)<\infty \Leftrightarrow$ $E\left(\left|\min _{n \geqslant 0} \hat{S}_{n}\right|^{\alpha}\right)<\infty$, so the equivalence of (1.31) and (1.33) follows from Proposition 4.1 of [8].

Since $\lim _{t \rightarrow \infty} X_{t}=\infty$ a.s., the process $H^{\#}$ is a subordinator, $H^{(0)}$ say, killed at an independent exponential time $\tau$, and its final value $H_{\tau}^{(0)}$ coincides with $\left|I_{\infty}\right|$ see [1, p. 172]. It follows that

$$
\begin{gathered}
E\left(\left(H_{r}^{\#}\right)^{\alpha} ; H_{r}^{\#}<\infty\right)<\infty \text { for a fixed } r>0 \Leftrightarrow E\left(H_{r}^{(0)}\right)^{\alpha}<\infty \\
\Leftrightarrow E\left(H_{r}^{(0)}\right)^{\alpha}<\infty \text { for all } r>0 \Leftrightarrow E\left(H_{\tau}^{(0)}\right)^{\alpha}<\infty .
\end{gathered}
$$

(One way to justify the last step is to relate the Laplace transform of $H_{\tau}^{(0)}$ to the Laplace transform of $H_{1}^{(0)}$ and then appeal to Lemma 16.) Thus (1.32) $\Leftrightarrow(1.33)$.

As for (1.34), first note that, on the event $T(-r)<\infty,\left|X_{T(-r)}\right|<\left|I_{\infty}\right|$. On the other hand, if $p=P(T(-r)<$ $\infty)$, then $\left|I_{\infty}\right|$ is stochastically dominated by $\sum_{1}^{N(p)} Z_{i}$, where the $Z_{i}$ are independent copies of $\left|X_{T(-r)}\right|$, given $T(-r)<\infty$, and $N(p)$ is an independent Geometric $(p)$ rv. Since $E\left(\left(\sum_{1}^{n} Z_{i}\right)^{\alpha}\right) \leqslant n^{\alpha} E Z_{1}^{\alpha}$, the reverse implication follows.

Finally, just as in the random walk case, we can show that for $t>r \geqslant 0$,

$$
P\left(\sup _{0 \leqslant t \leqslant \Lambda_{r}}\left|X_{t}\right|>t\right) \leqslant 2 P\left(\left|I_{\infty}\right|>t-r\right),
$$

and then argue just as in [8, p. 30], to see that (1.34), (1.35), and (1.36) are equivalent. 
Proof of Theorem 6. Since $g(a)>0$ and $T_{g}(r, a) \geqslant T_{r g(a)}$, the finiteness of $E\left(T_{g}(r)\right)^{1+\alpha}$ implies that of $E T_{r}^{1+\alpha}$ for any $r>0$ and $\alpha \geqslant 0$. Then use Theorem 1 .

Conversely, suppose $E T_{r}^{1+\alpha}$ is finite for some, hence every, $r>0$ and some $\alpha \geqslant 0$. Since $g \in R V(\rho)$ with $\rho<1$ as $t \rightarrow \infty$ we have $g(t) \leqslant t^{\eta}$ for $t \geqslant$ some $t_{0}(\eta)$ for some $\eta<1$. Select such an $\eta<1$, then $r g(t+a) \leqslant r(t+a)^{\eta}$ for $t \geqslant t_{0}$. Take $t_{0} \geqslant 1$ without loss of generality. Now $\lim _{t \rightarrow \infty} X_{t}=\infty$ a.s. implies $\lim _{t \rightarrow \infty} X_{t} / t=\mu \in(0, \infty]$ a.s. Thus $\liminf _{t \rightarrow \infty} X_{t} / t>0$ a.s. Hence we can find a $\delta>0$ with $P\left(X_{t}<\delta t\right.$ i.o. for $\left.t \rightarrow \infty\right)=0$. We can take $\delta>0$ smaller if necessary and then find an $r_{0}(r, a, \delta)>0$ so that the straight line with intercept $r_{0}$ and slope $\delta$ lies entirely above the function

$$
r\left(t_{0}+a\right)^{\eta} \mathbf{1}_{\left\{t<t_{0}\right\}}+r(t+a)^{\eta} \mathbf{1}_{\left\{t \geqslant t_{0}\right\}} .
$$

Since $g(\cdot)$ is nondecreasing, this function in turn lies entirely above $r g(t+a)$, for all $t \geqslant 0$. Now define

$$
T_{r_{0}}^{\delta}=\inf \left\{t>0: X_{t}>r_{0}+\delta t\right\} .
$$

Then $T_{r_{0}}^{\delta} \geqslant T_{g}(r, a)$, so it will suffice to prove that $E\left(T_{r_{0}}^{\delta}\right)^{1+\alpha}<\infty$. To see this, let $X^{(\delta)}=\left\{X_{t}^{(\delta)}, t \geqslant 0\right\}$ where $X_{t}^{(\delta)}=X_{t}-t \delta$; this Lévy process satisfies $P\left(X_{t}^{(\delta)}<0\right.$ i.o. for $\left.t \rightarrow \infty\right)=0$, thus $\lim _{t \rightarrow \infty} X_{t}^{(\delta)}=\infty$ a.s. Also we have

$$
T_{r_{0}}^{\delta}=\inf \left\{t>0: X_{t}^{(\delta)}>r_{0}\right\},
$$

so $T_{r_{0}}^{\delta}$ is the $T_{r_{0}}$ for $X_{t}^{(\delta)}$. Thus, by Theorem 1 (cf. (1.14)), $E\left(T_{r_{0}}^{\delta}\right)^{1+\alpha}<\infty$ for each $\alpha \geqslant 0$ if and only if there is an $x_{0}>0$ with

$$
A^{\delta}(x)>0 \text { for } x \geqslant x_{0} \quad \text { and } \int_{\left(x_{0}, \infty\right)}\left(\frac{x}{A^{\delta}(x)}\right)^{1+\alpha}\left|\bar{\Pi}_{\delta}^{-}(d x)\right|<\infty,
$$

where $A^{\delta}(\cdot)$ and $\Pi_{\delta}(\cdot)$ are the $A(\cdot)$ and $\Pi(\cdot)$ for the shifted process $X^{(\delta)}$. This process has the same characteristics as $X$ but with $\gamma$ replaced by $\gamma-\delta$. Thus $\Pi_{\delta}(\cdot)=\Pi(\cdot)$, and

$$
A^{\delta}(x)=(\gamma-\delta)+\bar{\Pi}_{\delta}^{+}(x)-\bar{\Pi}_{\delta}^{-}(x)+\int_{1}^{x}\left(\bar{\Pi}_{\delta}^{+}(y)-\bar{\Pi}_{\delta}^{-}(y)\right) d y=A(x)-\delta .
$$

We have $A(\infty)>0$ (Lemma 13) and similarly $A^{\delta}(\infty)>0$, so $A^{\delta}(x) \geqslant a A(x)$ for some $a>0$ for all large $x$. Thus (2.28) follows from (1.14), and hence $E\left(T_{r_{0}}^{\delta}\right)^{1+\alpha}<\infty$.

Proof of Theorem 7. (i) Suppose $E T_{1}(r, a)<\infty$ for all $r>0$ and some $a>0$. Then $E T_{a}<\infty$, so $\lim _{t \rightarrow \infty} X_{t}=$ $\infty$ a.s. and (1.9) holds. If $E\left|X_{1}\right|<\infty$ and $\mu:=E X_{1}>0$ then $P\left(X_{t}>(\mu+\varepsilon) t\right.$ i.o. $)=0$ for all $\varepsilon>0$, so given $\delta \in(0,1 / 2)$ there is a $t_{0}(\delta)>0$ such that $P\left(\sup _{s \geqslant t}\left(X_{s} / s\right)>\mu+\varepsilon\right) \leqslant \delta$ for all $t \geqslant t_{0}$. We can also assume $t_{0}$ so large that $P\left(X_{t_{0}}<0\right) \leqslant \delta$. Now by Doob's inequality

$$
P\left(\sup _{0 \leqslant t \leqslant t_{0}} X_{t}>r a\right) \leqslant \sup _{0 \leqslant t \leqslant t_{0}} E\left|X_{t}\right| /(r a) \leqslant 1-\delta
$$

provided $r$ is large enough, $r \geqslant r_{0}\left(t_{0}(\delta)\right)$, say. Also choose $r>\mu+\varepsilon$. Now

$$
P\left(\sup _{t>0}\left(X_{t} /(t+a)\right) \leqslant r\right) \geqslant P\left(\sup _{0 \leqslant t \leqslant t_{0}} X_{t} \leqslant r a, \sup _{t>t_{0}}\left(\left(X_{t}-X_{t_{0}}\right) / t\right)<\mu+\varepsilon\right),
$$

because if the event on the right-hand side occurs, then $X_{t} \leqslant r a \leqslant r(t+a)$ for $t \leqslant t_{0}$, while also for $t>t_{0}$, $X_{t}<(\mu+\varepsilon) t+X_{t_{0}} \leqslant(\mu+\varepsilon) t+r a \leqslant r(t+a)$. Thus for $r>(\mu+\varepsilon) \vee r_{0}$, 


$$
\begin{aligned}
P\left(T_{1}(r, a)=\infty\right) & \geqslant P\left(\sup _{0 \leqslant t \leqslant t_{0}} X_{t} \leqslant r\right) P\left(\sup _{t>t_{0}}\left(\left(X_{t}-X_{t_{0}}\right) / t\right) \leqslant \mu+\varepsilon\right) \\
& \geqslant \delta P\left(\sup _{t>t_{0}}\left(X_{t} / t\right) \leqslant \mu+\varepsilon, X_{t_{0}}>0\right) \\
& \geqslant \delta(1-2 \delta)>0 .
\end{aligned}
$$

This contradiction shows that the first conclusion in (1.9) must hold, i.e., (1.39) holds.

Conversely, (1.39) implies $\lim _{t \rightarrow \infty} X_{t} / t=\infty$ a.s. Now we can equivalently write

$$
T_{1}(r, a)=\inf \left\{t>0: X_{t}>r(t+a)\right\}=\inf \left\{t>0: X_{t}^{(r)}>r a\right\},
$$

where $X_{t}^{(r)}=X_{t}-t r$. Thus $T_{1}(r, a)$ is $T_{r a}$ for $X_{t}^{(r)}$, and since $\lim _{t \rightarrow \infty} X_{t}^{(r)}=\infty$ a.s. we have $E T_{1}(r, a)<\infty$. This proves the equivalence with (1.39). By Theorem 1, the $T_{r a}$ for $X_{t}^{(r)}$ has a finite moment of order $1+\alpha$, or, by (2.29), the same is true for $T_{1}(r, a)$, if and only if (2.28) holds with $r$ in place of $\delta$. As we saw following (2.28) this is equivalent to (1.14), which in the present case is equivalent to (1.19).

(ii) Now suppose $E\left(T_{1}(r, a)\right)<\infty$ for some but not all $r>0$ and some $a>0$. Then $E T_{a}<\infty$ and (1.9) holds, and by part (i) of the proof it must be the case that $0<E X_{1} \leqslant E\left|X_{1}\right|<\infty$. Conversely this implies $E\left(T_{1}(r, a)\right)<\infty$ for $r<E X$ via (2.29) and the fact that $\lim _{t \rightarrow \infty} X_{t}^{(r)}=\infty$ a.s. when $0<r<E X$. If $r \geqslant E X_{1}$, $\liminf _{t \rightarrow \infty} X_{t}^{(r)}=-\infty$ a.s., so the $T_{r}$ for $X_{t}^{(r)}$ has infinite expectation, thus the same is true of $T_{1}(r, a)$ via (2.29).

Finally suppose $0<E X_{1} \leqslant E\left|X_{1}\right|<\infty$ and $0<r<E X_{1}$. Then $E\left(X_{1}^{(r)}\right)=E X_{1}-r>0$, so by Theorem 1, the $T_{r}$ for $X_{t}^{(r)}$ has a finite moment of order $\alpha+1$ if and only if the second condition in (1.18) holds, i.e., if and only if $E\left(X_{1}^{-}\right)^{\alpha+1}<\infty$.

\section{Acknowledgements}

We are grateful to Ms Angharad Bryn-Jones for a useful observation, and to a referee for pointing out some errors and obscurities in an earlier draft.

\section{References}

[1] J. Bertoin, An Introduction to Lévy Processes, Cambridge University Press, Cambridge, 1996.

[2] N.H. Bingham, R.A. Doney, Asymptotic properties of super-critical branching processes, I: The Galton-Watson process, Adv. Appl. Probab. 6 (1975) 711-731.

[3] N.H. Bingham, C.M. Goldie, J.L. Teugels, Regular Variation, Cambridge University Press, Cambridge, 1987.

[4] R.A. Doney, Stochastic bounds for Lévy processes, Ann. Probab., in press.

[5] R.A. Doney, R.A. Maller, Stability and attraction to normality for Lévy processes at zero and infinity, J. Theoret. Probab. 15 (2002) 751-792.

[6] K.B. Erickson, The strong law of large numbers when the mean is undefined, Trans. Amer. Math. Soc. 185 (1973) 371-381.

[7] S. Janson, Moments for first-passage and last-exit times, the minimum, and related quantities for random walks with positive drift, Adv. Appl. Probab. 18 (1986) 865-879.

[8] H. Kesten, R.A. Maller, Two renewal theorems for general random walks tending to infinity, Probab. Theory Related Fields 106 (1996) $1-38$.

[9] W.E. Pruitt, General one-sided laws of the iterated logarithm, Ann. Probab. 9 (1981) 1-48.

[10] B.A. Rogozin, Local behaviour of processes with independent increments, Theor. Probab. Appl. 13 (1968) $482-486$.

[11] K. Sato, Lévy Processes and Infinitely Divisible Distributions, Cambridge University Press, Cambridge, 1999.

[12] V. Vigon, Votre Lévy ramp-t-il?, J. London Math. Soc. 65 (2002) 243-256. 